agreement regarding i) the use of advanced analytics on specific clinical scenarios and ii) the critical aspects when using these applications on the frame of PC. The sample included professionals from different countries and disciplines with expertise on chronicity, geriatrics and/or PC. Consensus was defined as $>70 \%$ of subjects agreement.

Results At this time, fifteen experts have answered the questionnaire $(37.5 \%$ of whom invited to participate). $78.6 \%$ were women, $71.4 \%$ were working in a clinical setting $>15$ years as physicians (57.1\%), nurses (35.7\%) and psychologists (7.1\%) $80 \%$ of them considered that the strategy based on an automatic tool (BD based) combined with front-line healthcare staff is the best way for identification of patients who could benefit from early PC approach. They assessed as 'very' and 'extremely useful' the use of BD models on research applications, both as a population health management tool (clinical clustering) and as a tool to improve the prediction of an outcome risk. However, the need for clinical validation and the dearth of evidence of practical benefits are the main critical aspects on the implementation of these tools.

Conclusions The view of experts can contribute to guide BD applications on advanced stages of illnesses. The consensus about the opportunities and gaps on the implementation of these tools will support clinicians in decision making processes.

\section{EVALUATION OF THE PROCESS OF SENDING OUT OACC QUESTIONNAIRE TO PATIENTS, PRIOR TO THEIR FIRST PALLIATIVE CARE NURSE SPECIALIST VISIT/ APPOINTMENT, JUNE 2019}

Julie Gater, Davina Bell, Emer McKenna. Douglas Macmillan Hospice

10.1136/spcare-2020-PCC. 147

The OACC Assessment Questionnaire is sent out to all appropriate patients at telephone triage who are assessed as needing to be seen within 1-2 Weeks. The Questionnaire looks at IPOS as part of OACC.

This process allows for the Patients to have more time to review their symptoms and to think about their main problems of concern over the last week, along with any practical problems resulting from their illness, prior to their Palliative Care Nurse Specialist visiting them in their own home for their first holistic assessment.

By enabling an opportunity for the Patients to complete the Questionnaire themselves, it reduces reliance on Healthcare Professional Staff assisting them, and gives more autonomy/direction about key issues for them. It can also be used as a channel for Patients and Families to have some open conversation prior to their first visit/assessment, and to give insight into areas we will cover, which are different to a District Nursing assessment.

This review aimed to establish the benefit of sending out the Questionnaire. Also to examine if the action of recording data within the process was robust, and to identify any areas that could be improved.

A random sample of 34 patients was selected for retrospective review of activity from sending out the Questionnaire to examination whether this was completed by the patient or helped by the nurse to complete on the first visit, and scrutiny of the data recorded on Crosscare thereafter.
Overall, it was found that there was benefit of sending out the OACC Questionnaires with 50\% of Patients completing this prior their PCNS visit. However, following analysis of the data recorded, we established that we needed to revisit training, particularly for PCNSs for navigating the IPOS windows and recording data therein.

\section{PALLIATIVE CARE FOR HOMELESS PEOPLE: A SYSTEMATIC REVIEW OF SYSTEMATIC REVIEWS FOR AN EVIDENCE BASED CLINICAL GUIDELINE}

Anna Gray. King's College London and St Francis Hospice (Romford)

\subsection{6/spcare-2020-PCC.148}

Background Homeless people die much younger and have higher rates of ill-health compared to the general population. This systematic review of systematic reviews seeks to understand 1. views and preferences of homeless people regarding palliative care, 2. barriers and facilitators to homeless people accessing palliative care and 3. evidence for advance care planning interventions in palliative care for homeless people. This informed the development of a guideline for use by healthcare professionals providing palliative care for homeless patients.

Methods Searches took place of PubMed, Embase, PscyhINFO, CINAHL, Web of Science, Medline and Cochrane Library from inception to December 2019. The quality of the studies was assessed using SIGN Methodology checklist 1. The GRADE system was then used to assess the quality of the evidence and strength of the recommendations. Themes were identified following data analysis.

Results Five systematic reviews met the eligibility criteria. Three were rated as moderate quality, one as poor quality and one as unacceptable quality. Nine types of themes were then identified. First, views and preferences; homeless people recall poor experiences of healthcare in the past and have unique concerns regarding their health. Five barriers to palliative care were also identified, namely; lack of basic needs being met, having competing priorities, being ill-informed about their condition, hospice and hostel staff having insufficient knowledge, and poor co-ordination of care. The emerging facilitator was that homeless patients trust the staff they already know. Finally, many homeless patients benefit from advance care planning interventions, if they feel able to engage in the process. The quality of the recommendations was moderate to low. The strength of the recommendations was mostly strong.

Conclusions In this review, the views, preferences, barriers, facilitators, and interventions in palliative care for homeless people were established, informing a new clinical guideline.

\section{IMPLEMENTATION OF TREATMENT ESCALATION PLANS (TEP) AND DNACPR DECISIONS AT ST GEORGE'S UNIVERSITY HOSPITALS NHS FOUNDATION TRUST}

Amy Hawkins, Megan L Papworth. St George's University Hospitals NHS Foundation Trust

\subsection{6/spcare-2020-PCC. 149}

Introduction Treatment escalation plans (TEP) are a tool to promote advance care planning, appropriate decision making and good communication between professionals, patients and 